\title{
Cyber Health Psychology: The use of new technologies at the service of psychological well being and health empowerment
}

\author{
Pasquale Caponnetto, ${ }^{1}$ Michele Milazzo, ${ }^{2}$ \\ ${ }^{1}$ Departement of Clinical and Experimental Biomedicine, and ${ }^{2}$ Department of Education Sciences, \\ University of Catania, Italy
}

\begin{abstract}
Man and technology seem to co-evolve into a process of reciprocal conditioning. On the one hand, the man modifies (and evolves) the technology according to his needs, on the other the technology revolutionates the man and the way in which it lives. Psychology, therefore, as a discipline afferent to the human sciences, is called to take an interest in this relationship and to understand its complexity. A fundamental role in this sense is covered by "cyberpsychology" which investigates all those psychological phenomena that are associated with technology and aims to analyse the processes of change triggered by the interaction between man and the new media. From the psychological point of view, if on the one hand it is important to understand how man changes in contact with the new technologies and to what problems he is meeting. On the other hand, there is a need to understand how new technologies, given their transformative potential, can find a place within the therapeutic practice. In this regard, some of the technologies used in the clinical field have been analysed including: virtual reality, biosensors, artificial intelligence and affective computing. With the aim of understanding to what extent and how technological progress and the emergence of new technologies can contribute and generate value within the psychological panorama. Following the PRISMA statement a bib-
\end{abstract}

\footnotetext{
Correspondence: Pasquale Caponnetto, Departement of Clinical and Experimental Biomedicine, University of Catania, Catania 95123, CT, Italy.

E-mail: pcapon@unict.it

Key words: Cyberpsychology, Virtual reality, Avatar therapy, Artificial intelligence, Biofeedback in psychology.

Contributions: The authors equally contributed to this paper with conception and design of the study, and final approval of the final version.

Conflict of interest: The authors declare no potential conflict of interest.

Funding: None.

Received for publication: 12 September 2019.

Accepted for publication: 28 October 2019.

This work is licensed under a Creative Commons AttributionNonCommercial 4.0 International License (CC BY-NC 4.0).

$\left.{ }^{(}\right)$Copyright: the Author(s), 2019

Licensee PAGEPress, Italy

Health Psychology Research 2019; 7:8559

doi:10.4081/hpr.2019.8559
}

liographic research was carried out, which provided for consultation of the Medline and PsycINFO databases. The criteria according to which works have been selected rather than others refer to their precision and sensitivity with which they propose to treat technological applications in the field of health psychology and from this the emergence of the new theme "CYBER HEALTH PSYCHOLOGY". The results of the research suggest that the integrated use of psychological techniques and new technologies is extremely productive in terms of potential improvement of health and therefore of "health empowerment". In this vision, new technologies are not intended to replace traditional procedures but to integrate them by making available features and potential that man does not have in nature. Given the great potential of the instruments analyzed that still today continue to evolve and refine it is advisable to know them, validate their effectiveness and adapt our operational models to new realities.

\section{Background}

We live in a world where change is always quicker. One of the factors that more than others influences the rapidity of this phenomenon is technological progress. Technology is revolutionating the lifestyle of the person, the way in which he communicates and relates to others and to himself (Riva, 2008).

Nowadays most of the interactions that young people have between them involve the use of technological devices. Cognitive sciences have widely demonstrated that the repeated use of a given device over time alters the individual's cognitive system. The technology can no longer be considered merely as an object separable from the subject, as it has become part of everyday life to such an extent as to constitute a kind of cognitive prosthesis with all the constraints and opportunities that this entails (Villani, Grassi, \& Riva, 2011). The fact that the relationship is increasingly shifted on the technological dimension obliges psychology to know and analyse this kind of relationship carefully. A fundamental role in this sense is covered by "cyberpsychology", whose field of investigation includes all the psychological phenomena associated with technology. It has as its objective the study and analysis of all those processes of change activated through the interaction between man and new technologies, in particular it focuses on the creation of new models of communication and interaction, which deviate Dramatically from the classic face-to-face modes (Moher, Tetzlaff, Altman, \& The PRISMA Group, 2009). From the psychological point of view, if on the one hand it is important to understand how man changes in contact with the new technologies and to what problems he is meeting; On the other hand, there is a need to understand how new technologies, given their transformative potential, can find a place within the therapeutic practice. In this regard we will analyse some of the technologies used in the clini- 
cal field including: Virtual reality, biosensors, artificial intelligence and affective computing; with the aim of bringing out how and in what way these can be used in the service of health.

\section{Core tips}

Some of the technologies used in the clinical-psychological field have been analysed, including: virtual reality, biosensors, artificial intelligence and affective computing; With the aim of understanding how technological progress and the emergence of new technologies can generate value within the psychological panorama. Research results suggest that the integrated use of psychological techniques and new technologies is extremely productive in terms of potential improvement of health and therefore of "health empowerment". Given the great potential of the instruments analyzed that still today continue to evolve and refine it is advisable to know them, validate their effectiveness and adapt our operational models to new realities.

\section{Materials and methods}

Following the PRISMA statement (Moher et al., 2009) a bibliographic research was carried out, which involved the consultation of the Medline and PsycINFO databases. The research strategy involved the use of the following keywords: "Cyberpsychlogy" "Virtual Reality" - "Avatar Therapy" - "Artificial intelligence" "Biofeedback in Psychology". By narrowing the research field to the publications of the last ten years, only the researches that presented an abstract were taken into consideration, through which a first distinction and inclusion of the relevant topics to the reference track was made.

Furthermore, three texts and a scientific journal article were consulted outside the databases, as indicated in the diagram in Figure 1 among the secondary sources.

Criteria according to which works have been selected rather than others refer to their precision and sensitivity with which they propose to treat the technological applications in the clinical psychological field deriving from the encounter between new technologies and psychology.

In reference to the limiting research criteria, we have chosen to take into consideration only the last ten years of publications relating to the research field, as the subject matter follows a rapid evolution over time. However, the use of particularly outdated works could have been obsolete and not functional to the objective that instead aims this elaborate, that is to emphasize what innovation and technological progress can provide to Psychology to broaden its fields of application and increase its effectiveness.

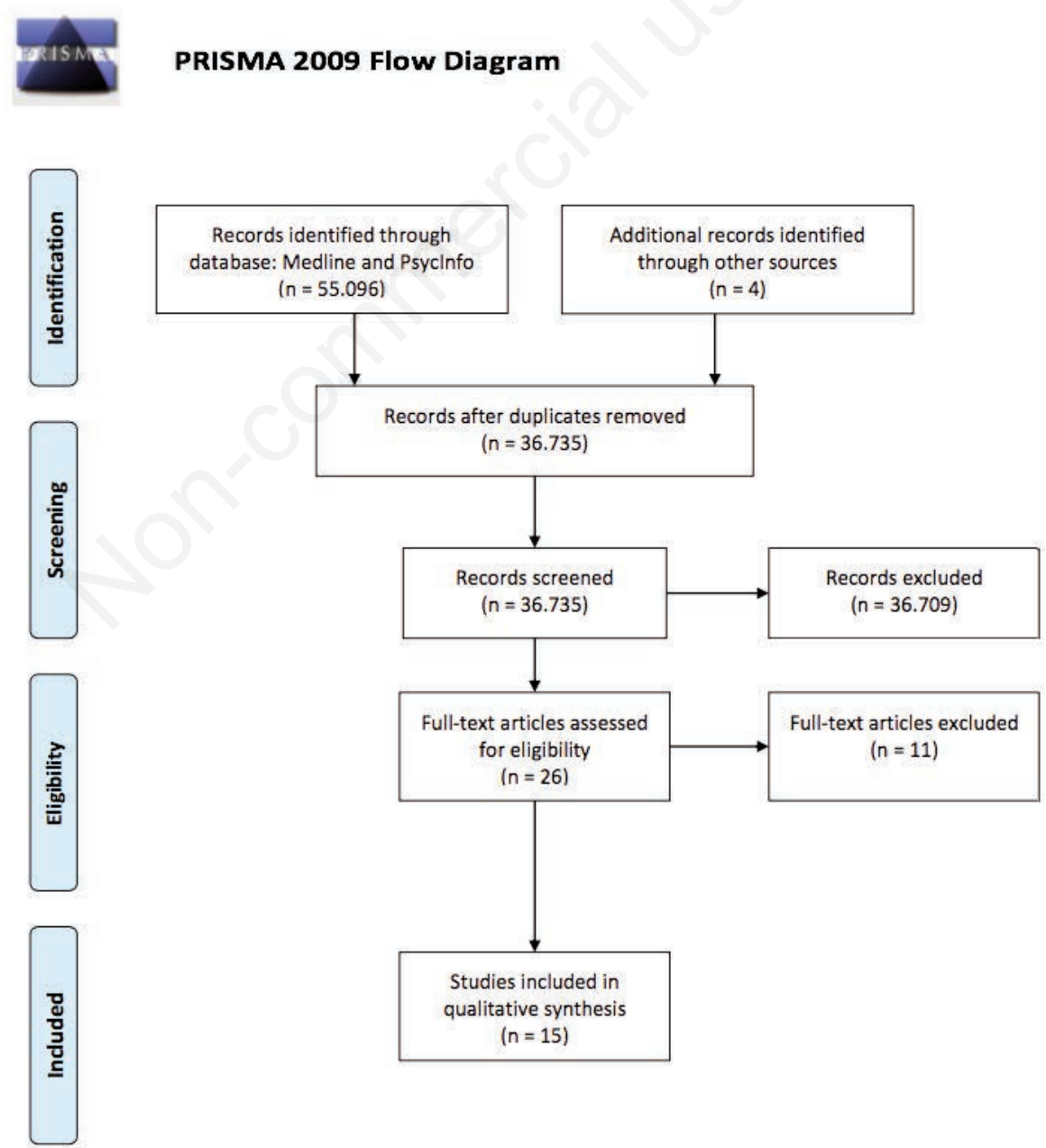

Figure 1. Flow Diagram. 


\section{Man and technology}

In the age of digitization, technology has become part of the everyday life of each of us, and invests a multitude of aspects: from work, to study, to relationships (Riva, 2018). It is not a factor that intervenes in an isolated way in the life of man, but rather of something that characterizes it and which modifies the environment in which it lives (Connolly et al., 2016). In the history of technology, a constant trend is identifiable: to make human-machine interaction as close as possible to that which man is accustomed to having in his own daily reality (Villani et al., 2011). The big companies that have been involved in technology have realized that the more the human-machine interaction was intuitive and at hand for man, the more this was inclined to introduce the use of the medium in their everyday life, favouring its diffusion. In order to interact with the first desktop computers, the man needed a technical knowledge that was not indifferent, so they were used only in the working environment; Later with the invention of technologies that made interaction more and more intuitive (such as the mouse or touch screen), the use of these tools has also spread among those who did not need it for work (Riva, 2008). The digitalization of the traditional media has led to the emergence of new media, more and more interactive and modular. New media allows subjects to interact with each other, overcoming the limits of face-to-face communication. In addition to overcoming the space-time limits, this type of mediated interaction modifies the structure of the relationship itself (Riva, 2008). Psychology in general tends to analyze this type of relationship highlighting the negative psychological implications, from psychopathologies to behavioral deviations such as: dependence on smartphones, video games, internet, cyberbullying, sexting etc.; however the relationship with the media is much more complex: from its analysis it is possible to understand how people represent themselves and the society in which they live; reducing the analysis to a direct linearity with what is negative does not allow to fully grasp all the aspects, both positive and negative that derive from it. In this regard, cyberpsychology is set in a proactive manner with respect to the traditional psychology of the media and tries to overcome that reactive nature for which only the "malus" of coexistence between man and the media were highlighted. The cyberpsychology investigates all those psychological phenomena that are associated with technology and has as its objective the analysis of the processes of change activated by the interaction between man and new media (Riva, 2008). The new media have created a new social space: cyberspace, an immaterial dimension, an unlimited communication space where individuals can interact with each other through the use of electronic tools such as computers, smartphones or any other tool that has the possibility to to connect in this "common space" (Riva, 2008). The ease and immediacy with which a person, thanks to this virtual space, can communicate with others have caused the shift of the relational dimension from the real to the virtual. Denying that the virtual exists is an illusion, the relational dimension of Man is composed of the totality of his relationships, both those that entertain in reality and those that have origin and development through virtual (Riva, 2008). Man is a social being, and if on the one hand many see technology as a cold tool of destruction of the socialization of the individual, on the other some see in technology a support tool that expands and integrates the social dimension of Subject (Riva, 2008). Beyond the point of view from which we observe this phenomenon there is however a given objective: the virtual cannot be judged in an isolated way compared to the "real". Man in the age of digitalization and new media is immersed in a dimension in which what happens in reality flows on the virtual and vice versa; however, the understanding of how one configures and constructs the relational dimension of the individual cannot fail to pass from a careful analysis of the continuous interweaving between his online and offline life (Riva, 2008). Cognitive sciences have amply demonstrated how the use of a given device alters the individual's cognitive system and in a certain sense extends the corporeity of the subject. Technology has basically become a kind of cognitive prosthesis, a direct extension of the mind by which we organize and grasp information deriving from the environment, with all the constraints and opportunities that derive from it (Villani et al., 2011). In this background there is a question to which different theoretical visions, compared to technology and new media, have tried to answer: are we to change the media or are the media to change us? In response to this question to one extreme we find the technological determinism of McLuhan and on the other the social costructionism of Williams (Riva, 2008). According to McLuhan the use of a medium (the instrumentation that mediates the interaction between two individuals) not only modifies the interactions, but also affects the way of feeling and thinking of those who use it, thus configuring itself as the main tool of change Social. According to Williams, however, the media have a psychosocial origin and arise from the need to achieve a socially defined goal, thus configuring itself as a final product of the social push. So, if on the one hand it seems that man has direct and conscious control of the media, on the other it seems that the media change the ability of the individual in an unpredictable way as a function of a psychobiological adaptation. Trying to integrate these two visions it is possible to say that if on the one hand the new media and new technologies are conceived according to a goal defined a priori, with the purpose of performing certain functions, on the other it is also true that at the time when these Technologies become part of man's life exert profound changes both in its being and in its environment (Figure 2); However, a circular vision in which man and technology co-evolve in a reciprocal way is more functional in the comprehension of this complex phenomenon (Riva, 2008).

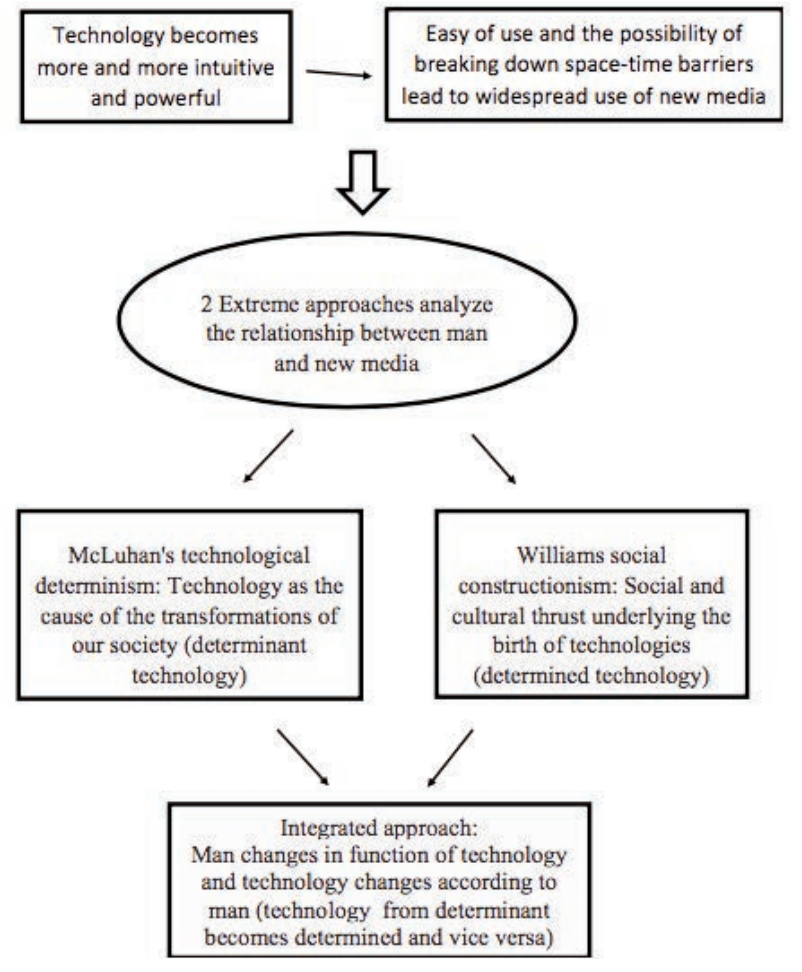

Figure 2. Relationship between man and technology. 


\section{From cyberpsychology to cyber health psychology}

The widespread use of new technologies obliges all health sciences and therefore also psychology to know and analyse them carefully. From the psychological point of view, knowing the dynamics of reciprocal change between man and technology is important to understand risks and opportunities (Riva, 2018). In the last two decades important advances have been made on the scientific study of well-being, and also the concept of health over the years has undergone considerable changes (Villani et al., 2011). "Health" has been defined by the World Health Organization in 1948 as: “... a state of complete physical, mental and social wellbeing and does not consist only in an absence of sickness or infirmity...". Health disciplines therefore should not only focus their attention on disease and treatment but also have to be interested in the activation of health promotion and maintenance pathways. In particular, in the psychological field, the study of this concept has given rise to positive psychology, which aims to study and intervene primarily on the sources of well-being rather than those of malaise, emphasizing the fundamental role of the individual's resources and potentials, stimulating a personal attitude that allows the psychological resources to be oriented in a constructive way in the tasks and difficulties of life (Villani et al., 2011). Martin E.P. Seligman has been recognized as the father of positive psychology and he maintains that psychology must pay equal attention both to the pathological aspects that concern the person, and to the positive and proactive aspects of human existence (Table 1).

Wellbeing in this sense can be analyzed by two fundamental points of view: objective indicators and subjective components (Villani et al., 2011). The objective elements refer to: income, social status, physical health conditions and housing conditions; however, stopping at an analysis of the objective components is not sufficient to delineate the general well-being of an individual, since all those subjective components that are relevant to describe the complexity of the phenomenon are missing (Villani et al., 2011). The subjective components refer to the assessments that the individuals themselves provide of their own state of health, of their degree of satisfaction, of the general well-being perceived. What a subject think about his well-being affects his condition of general health (Villani et al., 2011). In this perspective it becomes important, therefore, to understand what are the variables that influence this perception and how it is possible to intervene to modify them positively. A concept of particular relevance in this sense is that of Health Empowerment which refers to that process of active strengthening of one's well-being and adaptation to the environment (Villani et al., 2011). The purpose of this process is: to facil- itate change and to help the person get out of a static situation; this change can be solicited on different levels: emotional, relational, social, motivational. Going to work on these dimensions, stimulating them positively, it is possible to change the quality of the wellbeing perceived by the individual and therefore to control that subjective component which is an integral part of the overall wellbeing of the subject.

Given the transformative potential of technology to man, it would be interesting to understand if this potential could be exploited to change, in a targeted and controlled way, certain aspects of his life in order to improve his well-being condition. Positive psychology provides a good theoretical framework for understanding how new technologies can be put at the service of health.

Positive Technology (TP) is an emerging discipline that has focused on this theme and is defined by Riva in 2012 as: "a scientific application approach that uses technology to change the characteristics of our personal experience - structuring, increasing or replacing it with synthetic environments - in order to improve the quality of our personal experience and increase the well-being in individuals, organizations and companie".

\section{Technologies at the servicee of health}

"Psychology has always been a relational activity, in which the thrust to change and reflection is born within the profound communication between psychologist and user" (Riva, 2008). Within this communicative process the ability to understand the emotion of others, the emotional competence, and the relational capacity occupy an important space. Reflecting on these characteristics it is difficult to think that technology, given its "cold" and purely instrumental nature, can make its contribution to that effect.

Is it possible to think that emotion and affectivity can become part of the world of computer technology?

Since the end of the Nineties researches have been carried out that have led to the realization of machines able to recognize and model emotional information (affective computing), with the attempt to overcome the idea of a cold technology and Detached, towards a conception according to which a computer is transformed from mere "instrument, object" to a real "social actor", where man and machine are within a system of reciprocal interaction (Villani et al., 2011). Hudlinka in 2003 identifies four macroareas of study, useful to orient themselves in the research panorama defined Affective Human Computer Interaction:

1. Detection and recognition of emotions: the measurement of psycho-physiological indexes, the recognition of facial expres-

Table 1. From psychology to positive psychology. Psychology tries to understand the causes of: Positive sychology tries to understand the causes of:

Pain

Happiness

Stress

Positive emotion

Anxiety

Health

Depression

Resiliency

Unhappiness

Equilibrium

Deviance

Involvement

Anger Achievement 
sions, vocal profiling and stereotypical gestures allow the machine to initiate a process of processing Information that leads to the detection and recognition of the user's affective status.

2. Adaptation to the user's affective status: Once the user's affective condition is identified, the machine will have to respond in an adaptive and functional way to the predetermined objectives.

3. Expression of emotions and affective status: The machine is programmed to be able to express emotions to facilitate the communicative process, exploiting verbal and non-verbal modalities.

4. Architectures and emotional-cognitive models: This research area refers to the design of cognitive-emotional architectures that allow the adaptive functioning of the agents in terms of emotional-cognitive competence.

Despite the many potential that this area of research offers in different fields, from the medical, to the educational or commercial, it is possible to notice a suspicious and negative reaction to affective computing, as if one feared the occupation by the Machine of a space of competence that has always been exclusive of human nature (Villani et al., 2011).

In fact, a more thorough analysis can not help but see the many advantages offered by the meeting between new technologies and psychology.

\section{Virtual reality}

Virtual Reality (VR) is an application that allows users to move and interact in real time in a virtual environment generated by a computer. VR systems can generally be grouped into two categories: immersive and non-immersive systems. In non-immersive virtual reality, the virtual environment is shown through monitors and interaction with it is done by mouse and keyboard. In The psychological field the most used type of virtual reality is the "immersive" (IVR) (Villani et al., 2011). The factors that distinguish the IVR compared to other audiovisual applications are: the perception of being immersed in a virtual environment and a strong interactivity (Bouchard, 2011). The helmet/visor has inside the position sensors that record and encode the user's displacement and communicate it in real time to the computer which, through a graphical elaboration, reproduces the visual consequent to the displacement carried out. This process gives the user the illusion of being immersed within the virtual environment. The use of headphones/earphones in combination with the viewer increases this type of perception by providing the user, in addition to the visual component, a hearing experience. The degree of immersiveness perceived by the user is proportionate to the degree to which all perceptive senses of the person are adequately involved, therefore the auditory component is relevant to this purpose. The joystick/wired gloves are the tool through which the user has the ability to interact and modify the surrounding environment; Through the use of these devices it is detached from the position of mere passive observer becoming active component and responsible for the changes within the virtual space in which it is found catapulted (Villani et al., 2011). The VRI configuration can also include instruments such as cybertute that provide the user with a feed-back in terms of touch, weight and strength (Mitrusia \& Giotakos, 2016).

The purely experiential nature of this type of application makes it particularly useful in the psychological treatment of different problems. In recent years several studies have demonstrated the efficacy of VR in the psychotherapeutic field in the treatment of phobias (Mitrusia \& Giotakos, 2016), social anxiety disorder (Anderson et al., 2013), post-traumatic stress disorder (Botella et al., 2015), panic disorder (Perez-Ara, 2010)

The advantage of using this technology is the possibility of creating an ad hoc and tailor-made environment for the patient, where the therapist has total control over the variables at stake. Imagining that we have to set up and deal with a psychotherapeutic pathway with a phobic patient, so we need to apply an exposure therapy, the advantage that this type of application offers is the possibility of adapting the phobic stimulus to characteristics of the patient adjusting the levels of intensity, and then gradually evolving the session in session in a functional way to progress of the patient's abilities (Villani et al., 2011). In this situation the advantages compared to an in vivo exposure are evident. The virtual allows to create situations that in vivo would be difficult to manage and implement in a study/laboratory. Moreover, the control of the variables exercised by the therapist drastically reduces the risks associated with the unpredictability of effects that may occur in an in vivo exposure (Villani et al., 2011).

The meta-analysis conducted by Morina N. And colleagues highlighted how the results obtained during VRET (virtual Reality Exposure Therapy) in virtual environments are generalizable and can be reported to real life. Analyzing the behavior of patients before and after the VRET there has been a significant positive change in terms of response to phobic stimulus. Moreover, the results of the analysis do not reveal significant differences between VRET and in vivo exposure in terms of efficacy (Morina et al., 2015).

In this perspective it is possible to identify a sort of bridge between "reality" and "virtual reality" that are communicating with each other. The basis of this communication is a common language identifiable in the concept of 'experience'. The analysis of the experience, beyond the theoretical approach of reference, is configured like that common ground on which one works in a psychotherapeutic path, for this reason the IVR, configuring itself as an extremely experiential instrument, is functional (Villani et al., 2011). Traditionally, the cognitive behavioral approaches of exposure therapy, beyond the in vivo exposure, also include procedures that use the imagination of the patient; In this type of setting it must relax and imagine, under the guidance of the therapist, the stimulus that gradually progresses and becomes increasingly frightening (Villani et al., 2011). The relevance of this methodology is not questioned despite it use a reality imagined (with the limits that it entails), because at the center of the activity of analysis there is the experience and how this is lived also simply through an imagination. Among the limitations of this technique, however, is the fact that the patient may be unable to recreate the scenarios through the imagination due to the pathological avoidance of problematic memories, as often happens in post-traumatic stress disorder (PTSD) (Mitrusia \& Giotakos, 2016). The use of VR in this case could exceed this limit, as it allows the patient a realistic three-dimensional visualization of the dreaded situation (Villani et al., 2011).

The virtual reality has also attracted the interest of positive psychology, which, concentrating on strengthening the well-being and the causes associated with it, investigates its applicability. Studies in line with this perspective have observed how: "individuals interacting in environments enriched with a variety of positive visual and auditory stimuli report a great improvement in the levels of self-efficacy and emotional state"(Villani et al., 2011). Later Valtchanov and colleagues demonstrated that the RV immersion in a "natural" environment generated by a computer can affect the stress levels perceived by the user, increase the positive sensations and reduce negative (Valtchanov, Barton, Ellard, 2010). This suggests that virtual reality can also be used to manipulate experiences 
related to self-efficacy and emotions.

The multitude of advantages and applications that the virtual reality brings out is accompanied however by difficulties and limits to which it still can not easily escape. Until a few years ago the cost of the instrumentation needed to put in place a functional therapeutic setting of virtual reality immersive (IVR) was absolutely out of reach by the economic availability of those who exercised the profession (Mitrusia \& Giotakos, 2016). Fortunately, with the passing of time and the evolution of technology, the cost of these instruments decreased dramatically, becoming accessible from the past (Mitrusia \& Giotakos, 2016). Beyond the cost of instrumentation, another difficulty concerns the need for specific technical skills and the hardware and software knowledge of the instruments to be used (Mitrusia \& Giotakos, 2016). Knowledge that often, just in the psychological field, are not easy to understand for those who work in the field. From the physiological point of view, in the end, slight and temporary side effects are recorded such as nausea, dizziness or headaches following the VRET (Mitrusia \& Giotakos, 2016).

\section{Avatar Therapy: an example of virtual reality in the therapeutic field}

Julian Leff and colleagues (2014) have developed a new type of therapy, addressed to patients suffering from schizophrenia with persecutory auditory hallucinations, which involves the use of a non-immersive virtual reality.

Through the use of a software the patient is responsible for creating an avatar (virtual representation) of the entity, human or not, from which it feels persecuted. The creation of the Avatar foresees both the visual appearance (choice of the conformation of the face) and the auditory aspect (choice of the voice tone).

The setting allows the patient to be seated in a room in front of a monitor on which the avatar he creates appears. The therapist sits in an adjoining room and through a computer connected to the patient monitor takes control of the Avatar. What the therapist says is replicated by the avatar with the voice that the patient has previously chosen. The therapist through the computer can choose to report something to the patient using his own voice or to make the avatar speak by impersonating the persecutory entity.

Patients are asked to enter into a dialogue with their avatar and are encouraged to oppose it. The therapist has the opportunity to see the patient and listen to what he says so that he can regulate his intervention according to the recorded reactions. An important component of the therapy is that after the first two or three sessions the Avatar (controlled by the therapist) ceases to be violent and persecutory, becoming more and more supportive with the patients, complimenting them and praising their good qualities, changing Progressively even at the visual level becoming more smiling and friendlier. Each session is recorded and the audio file is transferred to a personal media player that is delivered to the patient and then used at any time to reinforce control over the persecutory "voice".

The main purpose of the therapy is to facilitate the dialogue with the avatar so that the patient can learn to resist the persecutory entity that he represents to control it and that it is generalized outside the virtual context.

The results of the study conducted by Julian Leff seem to be very encouraging. Many of the patients treated with Avatar therapy declare a significant reduction in intensity and frequency about auditory hallucinations, and greater control over their occurrence. In some cases, there is also the total disappearance of the "voices" verified after several follow-up (Leff et al., 2014).
This procedure has also brought significant results on those who could not control the phenomenon through pharmacological therapies (Leff et al., 2014).

\section{Biosensors: biofeedback and neurofeedback}

In the medical and psychological practice particular biosensors are used, able to measure a multitude of parameters. Biofeedback is a tool that is used to measure and monitor the physiological reactions of a patient: heartbeat, respiration, blood pressure, brain activity and muscle tension (Figure 3). Within the psychological research, biofeedback assumes a function that goes beyond the "measure " becoming a real technique,]aimed at teaching users to have awareness about their physiological changes, Increasing its control capacity in particular situations (Villani et al., 2011). The measurements made by biosensors are made known in real time to the user of the biofeedback through acoustic or visual (Biondi \& Valentini, 2014). Signals (visual or auditory) represent the mirror of the user's internal state; Mirror through which he learns to recognize himself and control himself (Figure 3). During the biofeedback therapy, the patient also teaches relaxation and stress management techniques (Biondi \& Valentini, 2014).

The use of biofeedback has also been associated with research into applied psychology in virtual reality (Villani et al., 2011). The integrated use of the two instruments (VR + biofeedback) allowed the patient to become aware of their physiological modifications through a direct action/modification on the virtual environment in which it was inserted (Villani et al., 2011). Real-time communication between biofeedback and virtual reality software produced an adaptive change in the environment compared to the user. The use of biofeedback is effective in the treatment of anxiety and stress disorders (Biondi \& Valentini, 2014). The same principle has also been applied with another type of biosensor: Neurofeedback. This particular sensor allows to record the cerebral activity of the central nervous system, by performing an electroencephalogram (EEG). The recorded data is simultaneously transmitted in a screen, providing a graphical representation of the brainwaves. "The aim of this treatment is to teach participants how to change the response of their brainwaves in certain situations or contexts, focusing their attention on the proposed graphical representation
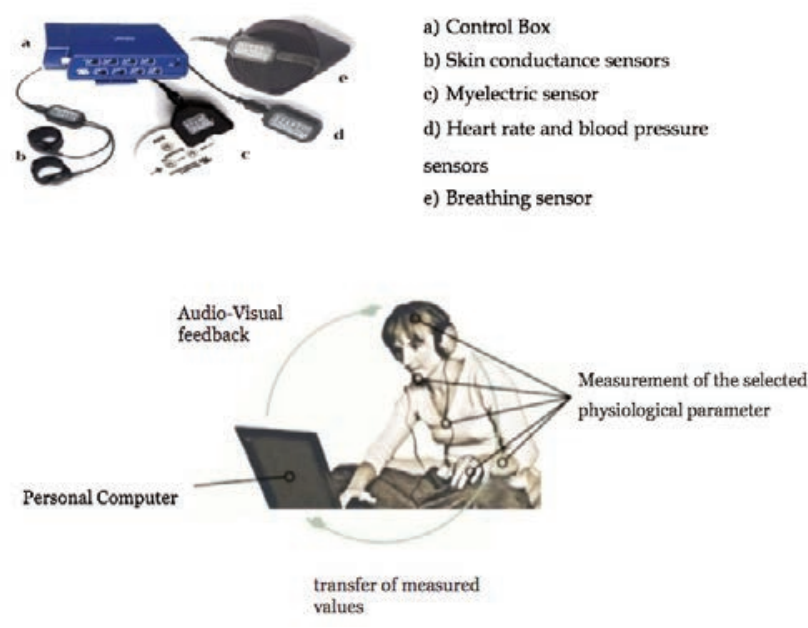

Figure 3. Biofeedback instrumentation and operation. 
and trying to Change it through attentional strategies and conscious monitoring" (Villani et al., 2011). The use of neurofeedback is effective in the treatment of attention and hyperactivity disorders, depressive disorders and learning disabilities (Villani et al., 2011).

In This vision, the integrated use of tools and techniques that foresee the encounter between the "real" and the "virtual", seem to be effective in order to modify the experience of the subject.

\section{Artificial intelligence}

In a rapidly evolving digital world, it is crucial that general practice embraces the potential of artificial intelligence (AI) and virtual reality (VR) in a real and proactive manner.

Artificial intelligence can be defined as the ability of a technological system to "act" autonomously, solving problems or carrying out tasks and activities typical of the mind and human abilities. The learning and management of information autonomously take place through the use of techniques such as "machine learning", "deep learning", and "Cognitive computing" (Beilby, 2018).

The term "machine learning" refers to a technique with which the software analyzes the data, recognizes its characteristics and "learns" from their comparison; The machine is able to establish links between the data and make predictions without having been previously programmed to accomplish these specific activities (Beilby, 2018). The techniques of "deep learning" manage to put the machine in the conditions of being able to simulate the functioning of a human brain using several levels of artificial neuronal networks, which generate automatic forecasts according to the data sets of Training provided to the machine (Beilby, 2018). "Cognitive computing" in the end represents the cognitive component of the machine, through which it is able to recognize images, sounds and language imitating the capacities of human recognition.

IBM Watson is the best-known example of artificial intelligence of cognitive computation (Beilby, 2018). Through the analysis and interpretation of data this tool has the ability to learn, understand and provide evaluations. Given the considerable amount of data that this instrument can store and combine, it is evident how its application can provide a valuable contribution in the clinical field. Just think of a common procedure of clinical diagnosis; The amount of data that IBM Watson is able to consider in the evaluation process far exceeds that of a human physician. Watson therefore can be configured as a valid assistant in the diagnosis phase, being able to analyze many data could bring to the attention of the professional only the salient ones, decreasing the false positives and improving the whole process of care (Beilby, 2018).

Among the various applications of Watson, since 2016 has been added a specific tool for psychological evaluation: the "Personality Insight". "In practice, this instrument, starting from an unstructured text of about 500-1000 words, is able to give a personality profile based on the same variables of the Big Five Questionnaire" (Classic personality test used by psychologists) (Riva, 2018). With respect to the Big Five Questionnaire, Watson makes it possible to obtain an evaluation without the active intervention of the assessed, of which it is necessary a simple written text, and with significantly lower costs and implementation times (Riva, 2018).

In this perspective, the use of artificial intelligence as Watson does not replace man, but it exalts its effectiveness and intelligence.

\section{Conclusions}

Men and technology seem to co-evolve into a process of reciprocal conditioning. On the one hand, the man modifies (and evolves) the technology according to his needs, on the other the technology revolutionates the man and the way in which it lives. Psychology, therefore, as a discipline afferent to the human sciences, is called to take an interest in this relationship and to $<<$ understand its complexity. Often, in the psychological field, the technology assumes a negative connotation and are highlighted the criticalities and the risks deriving from the use, but leaving out any advantages and opportunities that this could offer if used in a way Functional. The task of this elaborate was precisely to analyse some of the technologies that are currently applicable in the Psychotherapeutic field.

The research carried out suggests that the integrated use of psychotherapeutic techniques and new technologies is extremely productive in terms of potential health improvement and hence "health empowerment". In this vision the new technologies do not intend to replace the traditional procedures but to integrate them making available the characteristics and potentialities of which man does not have in nature. The challenge, however, is to understand the opportunities of the medium, (beyond its common use) and to decline its use according to its objective. Given the great potentialities of the instruments analysed that still continue to evolve and improve, it is advisable to be open and ready to adapt the operative models to the new realities.

\section{References}

Anderson, P., Price, M., Edwards, S., Obasaju, M., Shmertz, S., Zimand, E., \& Calamaras, M. (2013). Virtual reality exposure therapy for social anxiety disorder: a randomized controlled trial. Journal of consulting and clinical Psychology, 81(5), 751-760.

Beilby, J. (2018). Workforce innovation: Embracing emerging technologies. Australian Journal of General Pratice, 47(8), $522-524$.

Biondi, M., \& Valentini, M. (2014). Relaxation treatments and biofeedback for anxiety and somatic stress-related disorders. Rivista di psichiatria, 49(5), 217-226.

Bouchard, S. (2011). Could virtual reality be effective in treating children with phobias. Expert Review of Neurotherapeutics, 11(2), 207-213.

Botella, C., Serrano, B., Banos, R., \& Garcia-Palacios, A. (2015). Virtual reality exposure-based therapy for the treatment of post-traumatic stress disorder: a review of its efficacy, the adequacy of the treatment protocol, and its acceptability. Neuropsychiatric Disease and Treatment, 11, 2533-2545.

Connolly, I., Palmer, M., Barton, H., \& Kirwan, G. (2016). An introduction to Cyberpsychology. Cyberpsychology, behavior and social networking, 19(4), 294-295

Leff, J., Williams, G., Huckvale, M., Arbuthnot, M., \& Leff, A.P. (2014). Avatar therapy for persecutory hallucinations: what is itand how does it work? Psychosys; 6(2), 166-176.

Mitrusia, V., \& Giotakos, O. (2016). Virtual reality therapy in anxiety disorders. Psychiatrik 27(4), 276-276.

Moher, D., Tetzlaff, J., Altman, D., \& The PRISMA Group., (2009). Preferred Reporting Items for Systematic Reviews and Meta-Analyses: The PRISMA Statement. PLoS Med, 6(7)

Morina, N., Ijntema, H., Meyerbröker, K., \& Emmelkamp, P. 
(2015). Can virtual reality exposure therapy gains be generalized to real-life? A meta-analysis of studies applying behavioral assessments. Behaviour Research and Therapy, 74, 1824.

Riva, G. (2008). Psicologia dei nuovi media. Bologna: Il Mulino. Riva, G. (2018). Gli psicologi del futuro: il ruolo crescente delle nuove tecnologie. Psicologia contemporanea, p. 34-39.

Villani, D., Grassi, A., \& Riva, G. (2011). Tecnologie Emotive. Nuovi media per migliorare la qualità della vita e ridurre lo stress. Milano: LED Edizioni universitarie.

Perez-Ara, M., Quero, S., Botella, C., Banos, R., Andreu-Mateu, S., Garcia-Palacios, A., \& Brèton-Lopez, J. (2010). Virtual reality interoceptive exposure for the treatment of panic disorder and agoraphobia. Studies in health technology and informatics, 154, 77-81.

Valtchanov, D., Barton, K., \& Ellard, C. (2010). Restorative effects of virtual nature settings. Cyberpsychology, behavior and social networking, 13(5), 503-512. 